\title{
Cost-effectiveness of ambulatory oxygen in improving quality of life in fibrotic lung disease: preliminary evidence from the Amb0x Trial
}

\author{
To the Editor:
}

Fibrotic interstitial lung diseases (ILDs) are chronic and often progressive conditions resulting in substantial impact on morbidity, health-related quality of life (HRQoL), and health system costs. Ambulatory oxygen $(\mathrm{AO})$ used during routine daily activities could lead to improved exercise performance, reduced symptoms and improved mobility in daily life. A UK prospective, multicentre, mixed method, randomised controlled crossover trial in patients with fibrotic ILD (AmbOx trial: NCT02286063), the first study on $\mathrm{AO}$ effects in daily life, reported improved HRQoL after 2 weeks of AO compared to no intervention, when measured by the King's Brief ILD (K-BILD) questionnaire [1-3]. Although AO is used in ILD, evidence supporting its health-economic impact is absent. Here, we evaluate the cost-effectiveness of $\mathrm{AO}$ in patients with ILD, using data collected alongside the AmbOx Trial.

AmbOx included adults with fibrotic ILD with isolated exertional hypoxia [1, 2]. Participants (mean age 67.9 years; $31 \%$ female) were randomised to receive either AO during routine activities of daily living $(n=41)$ or no intervention $(n=43)$ first. After a two-week period, the groups were crossed over to the alternative. AO for 2 weeks was associated with a significant improvement in total K-BILD score compared to no oxygen (mean difference adjusted for treatment order 3.7, 95\% CI 1.8 to 5.6).

For this economic evaluation, data were analysed on a complete case basis ( $n=74$; of the 76 participants completing AmbOx, two had missing K-BILD scores). We estimated resource use and costs incurred from a National Health Service perspective. Costs of $\mathrm{AO}$ were based on the number of cylinders used (median 2.75 cylinders per week, range $0-14)[1,2]$ and assumed use of one nasal cannula per participant $[4,5]$. Rental prices for oxygen cylinders (GBP 0.25 per day), refills (GBP 10.56 per refill) and delivery (GBP 16.90 per delivery) were not available from UK suppliers (commercial in confidence) and so were assumed based on online information for an Australian medical gas cylinder company [6]. As AO was not anticipated to have any effect on disease progression in the short-term, costs related to any unplanned health professional visits or hospital admissions were not included. The mean cost for AO for 2 weeks was estimated to be GBP 91.02 (95\% CI GBP 77.83-104.21) per participant. Costs are expressed in GBP at 2017 rates, after conversion using AUD 1 equivalent to GBP 0.608.

We estimated the incremental cost per unit improvement in total K-BILD score, the primary trial outcome [1]. A one-point improvement in K-BILD score over a 2-week period was estimated to cost an additional GBP 25.21 (bootstrapped 95\% CI GBP 15.21-69.48). Sensitivity analyses using the intention to treat cohort (with multiple imputation to adjust for missing values) gave a similar incremental cost of GBP 27.38 (95\% CI GBP 15.68-86.77). Likewise, the estimated cost-effectiveness was not substantially impacted if the costs of the intervention were changed by up to $\pm 80 \%$ of that assumed in the primary analysis (mean estimated cost per unit improvement in K-BILD GBP 14.42-46.72 over a 2-week period).

We also undertook post hoc analyses to estimate the cost-effectiveness of $\mathrm{AO}$ in providing a benefit based on the number of participants who reported a minimum of an 8- or 4-unit improvement in total K-BILD

@ERSpublications

Ambulatory oxygen may be cost-effective in improving quality of life in fibrotic lung disease. To be more conclusive, we need to understand societal willingness to pay for quality of life improvements and whether improvements are sustained. http://bit.ly/2pAiBJi

Cite this article as: Whitty JA, Rankin J, Visca D, et al. Cost-effectiveness of ambulatory oxygen in improving quality of life in fibrotic lung disease: preliminary evidence from the AmbOx Trial. Eur Respir J 2020; 55: 1901157 [https://doi.org/10.1183/13993003.01157-2019]. 
score (these values have been reported as the minimal clinically important difference [3, 7]), or an improvement in the patient-reported global assessment of change in breathlessness and walking ability at the end of each 2-week treatment period, one of the pre-defined major secondary trial outcomes [2]. An 8-unit improvement in K-BILD score was reported for 13 (17.6\%, number needed to treat (NNT) 5.85) and a 4-unit improvement for $27(36.5 \%$, NNT 2.81) of participants [2]. Given an incremental cost of GBP 91.02 (95\% CI GBP 77.83-104.21) per person treated for 2 weeks, it is estimated to cost an additional GBP 532.47 (95\% CI GBP 455.31-609.63) to achieve one additional "responder" of at least 8 units or GBP 255.77 (95\% CI GBP 218.70-292.83) to achieve one additional "responder" of at least 4 units on the K-BILD, over a 2 -week period.

Data on global assessment of change was available for 76 participants. More participants perceived an improvement in their walking ability $(51,67.1 \%)$ or breathlessness $(52,68.4 \%)$ after receiving oxygen as compared to no oxygen $(1,1.3 \%$ in each case). This corresponds to an NNT of 0.02 for one additional patient to perceive an improvement in walking ability or breathlessness, and an estimated incremental cost of GBP 1.82 (95\% CI GBP 1.56-2.08) over 2 weeks to achieve one additional person perceiving an improvement.

This economic evaluation is the first to examine the cost-effectiveness of AO in fibrotic ILD, often a devastating and progressive group of diseases with substantial impact on patients' HRQoL and limited treatment options. Whether or not $\mathrm{AO}$ is considered to be of acceptable cost-effectiveness depends on society's (unknown) willingness to pay for an improvement in K-BILD score or the global assessment measures. The analysis suggests a much lower number needed to treat (and therefore cost for one additional responder) to obtain a perceived improvement in breathlessness or walking ability according to the global assessment measures, than to achieve an improvement in HRQoL according to the K-BILD. This apparent responsiveness for the global assessment of change might be related to the comparative bluntness of the single item measure and the possible impact of non-blinding on the participants' self-reported perception of improvement.

There are limitations associated with our study. Costs for oxygen cylinders were assumed, although sensitivity analysis suggests this assumption did not substantially impact findings. The AmbOx trial was open label, had a relatively small sample size and a short duration of oxygen use (2 weeks), making it challenging to extrapolate the costs or benefits to ILD patients in the longer term. It is possible that people using $\mathrm{AO}$ would be more likely to access long-term oxygen therapy as they become accustomed to the idea of using oxygen, with yet unknown benefits and drawbacks. This analysis should be considered as indicative only, until data from a larger study with longer follow-up is available to support more conclusive assertions. Finally, lightweight oxygen cylinders were used for all patients to standardise the intervention and to allow for higher oxygen flow rates in patients with more severe exertional hypoxia [2], but further studies are needed to assess whether portable oxygen concentrators may be more beneficial for the subset with milder ILD.

We had intended to evaluate cost per quality-adjusted life year (QALY) by deriving QALYs using utility values from the existing medical literature for health states described by the K-BILD or the St George's Respiratory Questionnaire, another secondary outcome measure [1]. However, appropriate HRQoL data in ILD patients on which to base an estimate of QALY gain are not available. Further research to derive a preference-based utility index for the K-BILD instrument is required to support accurate assessment of the benefits of treatment targeting HRQoL in ILD. Moreover, future trials should consider collecting a preference-based measure of health (such as the EQ-5D-5L) as an outcome $[8,9]$.

Nevertheless, despite these limitations, this study is the first to provide an indication of the cost-effectiveness of $\mathrm{AO}$ for improving HRQoL outcomes in ILD. Further evidence for the long-term effectiveness of AO, conversion of HRQoL outcomes in ILD to QALYs, and societal willingness to pay for HRQoL improvements are required to ensure the benefits of $\mathrm{AO}$ are accurately captured in economic evaluation and can be interpreted in resource allocation decisions.

Jennifer A. Whitty ${ }^{1,2}$, Jenny Rankin ${ }^{1}$, Dina Visca $\oplus^{3,4,5}$, Vicky Tsipouri $^{4,5}$, Letizia Mori ${ }^{4,5}$, Lisa Spencer ${ }^{6}$, Huzaifa Adamali ${ }^{7}$, Toby M. Maher ${ }^{5}$, Nicholas S. Hopkinson ${ }^{5}$, Surinder S. Birring ${ }^{8}$, Morag Farquhar ${ }^{9}$, Athol U. Wells ${ }^{4,5}$, Piersante Sestini ${ }^{10}$ and Elisabetta A. Renzoni ${ }^{4,5}$

${ }^{1}$ Norwich Medical School, University of East Anglia, Norwich, UK. ${ }^{2}$ Collaboration for Leadership in Applied Health Research and Care (CLAHRC), East of England, UK. ${ }^{3}$ Division of Pulmonary Rehabilitation, Istituti Clinic Scientifici Maugeri, IRCCS, Tradate, Italy. ${ }^{4}$ Interstitial Lung Disease Unit, Royal Brompton Hospital, London, UK. ${ }^{5}$ National Heart and Lung Institute, Imperial College London, London, UK. ${ }^{6}$ Liverpool Interstitial Lung Disease Service, Aintree Chest Centre, University Hospital Aintree, Liverpool, UK. ${ }^{7}$ Bristol Interstitial Lung Disease (BILD) Service, Southmead Hospital, Bristol, UK. ${ }^{8}$ Centre for Human and Applied Physiological Sciences, School of Basic and Medical Biosciences, Faculty of Life Sciences nd Medicine, King's College London, London, UK. 'School of Health Sciences, University of East Anglia, Norwich, UK. ${ }^{10}$ Dept of Respiratory Medicine, Surgery and Neurosciences, University of Siena, Siena, Italy. 
Correspondence: Jennifer A. Whitty, Health Economics Group, Norwich Medical School, University of East Anglia, Norwich Research Park, Norwich, NR4 7JT, UK. E-mail: jennifer.whitty@uea.ac.uk

Received: 25 Jan 2019 | Accepted after revision: 14 Oct 2019

Acknowledgements: The study was supported by the NIHR Biomedical Research Unit at Royal Brompton and Harefield NHS Foundation Trust and Imperial College London. Members of the DMC: Joanna Porter, Chair; Sunita Rehal, independent statistician; John S. Wort. Members of the TSC: Stephen Durham, Vicky Tsipouri, Ira Jakupovic, Elisabetta Renzoni, Dina Visca, Voon Ong, Arnab Datta. We thank John Tayu Lee for providing input into the design of the economic evaluation component of the study. We also thank the RBH Research and Finance Office for their help in organising and supporting the infrastructure needed for the study, in particular Patrick Petterson and Alla Kashif. The AmbOx study is funded in full by the Research for Patient Benefit Programme National Institute for Health Research (Ref: PB-PG-0712-28073). Additional infrastructure support for the study is being provided by the Royal Brompton NIHR-funded Biomedical Research Unit.

Support statement: This manuscript presents independent research funded by the National Institute for Health Research (NIHR) under its Research for Patient Benefit (RfPB) Programme (Grant Reference Number PB-PG-0712-28073). The funder has no role in the trial design, collection, management, analysis or interpretation of data, writing of reports or submission for publication. Jennifer Whitty is supported by the National Institute for Health Research (NIHR) Collaboration for Leadership in Applied Health Research and Care (CLAHRC) East of England. The views expressed are those of the author(s) and not necessarily those of the NHS, the NIHR or the Department of Health. Funding information for this article has been deposited with the Crossref Funder Registry.

Conflict of interest: J.A. Whitty reports grants from Research for Patient Benefit Programme National Institute for Health Research (ref: PB-PG-0712-28073), during the conduct of the study. J. Rankin reports grants from Research for Patient Benefit Programme National Institute for Health Research (ref: PB-PG-0712-28073), during the conduct of the study. D. Visca reports grants from Research for Patient Benefit Programme National Institute for Health Research (ref: PB-PG-0712-28073), during the conduct of the study. V. Tsipouri reports grants from Research for Patient Benefit Programme National Institute for Health Research (ref: PB-PG-0712-28073), during the conduct of the study. L. Mori reports grants from Research for Patient Benefit Programme National Institute for Health Research (ref: PB-PG-0712-28073), during the conduct of the study. L. Spencer reports grants from Research for Patient Benefit Programme National Institute for Health Research (ref: PB-PG-0712-28073), during the conduct of the study. H. Adamali has nothing to disclose. T.M. Maher has, via his institution, received industry-academic funding from GlaxoSmithKline R and D and UCB, and has received consultancy or speakers fees from Apellis, AstraZeneca, Bayer, Biogen Idec, Boehringer Ingelheim, Galapagos, GlaxoSmithKline R and D, Indalo, Pliant, ProMetic, Roche, Samumed and UCB. N.S. Hopkinson reports grants from Research for Patient Benefit Programme National Institute for Health Research (ref: PB-PG-0712-28073), during the conduct of the study. S.S. Birring reports grants from Research for Patient Benefit Programme National Institute for Health Research (ref: PB-PG-0712-28073), during the conduct of the study; fees (to Kings College Hospital) for using KBILD from Boehringer Ingleheim, Roche, Galapogos and Novartis, outside the submitted work. M. Farquhar reports grants from Research for Patient Benefit Programme National Institute for Health Research (ref: PB-PG-0712-28073), during the conduct of the study. A.U. Wells reports grants from Research for Patient Benefit Programme National Institute for Health Research (ref: PB-PG-0712-28073), during the conduct of the study; personal fees for lectures and advisory board work from Boehringer Ingelheim, Roche and Bayer, outside the submitted work. P. Sestini reports grants from Research for Patient Benefit Programme National Institute for Health Research (Ref: PB-PG-0712-28073), and Royal Brompton and Harefield NHS Foundation and Trust, during the conduct of the study. E.A. Renzoni reports grants from Research for Patient Benefit Programme National Institute for Health Research (ref: PB-PG-0712-28073), during the conduct of the study; personal fees for lectures and advisory board work from Boehringer Ingelheim and Roche, personal fees for lectures from Mundipharma, outside the submitted work.

\section{References}

1 Visca D, Tsipouri V, Mori L, et al. Ambulatory oxygen in fibrotic lung disease (AmbOx): study protocol for a randomised controlled trial. Trials 2017; 18: 201.

2 Visca D, Mori L, Tsipouri V, et al. Effect of ambulatory oxygen on quality of life for patients with fibrotic lung disease (AmbOx): a prospective, open-label, mixed-method, crossover randomised controlled trial. Lancet Respir Med 2018; 6: 759-770.

3 Patel AS, Siegert RJ, Keir GJ, et al. The minimal important difference of the King's Brief Interstitial Lung Disease Questionnaire (K-BILD) and forced vital capacity in interstitial lung disease. Respir Med 2013; 107: 1438-1443.

4 National Health Service (NHS). Home oxygen therapy: NHS Choices 2017. https://www.nhs.uk/conditions/homeoxygen-treatment/ Date last accessed: 15 May 2018.

5 National Institute for Health and Care Excellence (NICE). Oxygen. https://bnf.nice.org.uk/treatment-summary/ oxygen.html Date last accessed: 15 May 2018.

6 Ritter Dental. Medical Gas Cylinders Special Pricing Facility From BOC Gases, 2017. www.ritterdental.com.au Date last updated: 12 October 2017.

7 Barker RE, Nolan CM, Delogu V, et al. The King's Brief Interstitial Lung Disease Questionnaire: response to pulmonary rehabilitation and minimal important difference. Am J Respir Crit Care Med 2017; 195: A2849.

8 Szentes BL, Kreuter M, Bahmer T, et al. Quality of life assessment in interstitial lung diseases: a comparison of the disease-specific K-BILD with the generic EQ-5D-5L. Respir Res 2018; 19: 101.

9 Diamantopoulos A, Wright E, Vlahopoulou $\mathrm{K}$, et al. The burden of illness of idiopathic pulmonary fibrosis: a comprehensive evidence review. Pharmacoeconomics 2018; 36: 779-807. 